\title{
Toward Anomaly Detection in IaaS Cloud Computing Platforms
}

\author{
Mingwei Lin ${ }^{1}$, Zhiqiang $\mathrm{Yao}^{1 *}$, Fei Gao ${ }^{1}$ and Yang $\mathrm{Li}^{1}$ \\ ${ }^{1}$ Faculty of Software, Fujian Normal University, Fuzhou 350108, China \\ yzqfnu@163.com
}

\begin{abstract}
In order to monitor the running status of IaaS cloud computing platforms, performance metric data are collected to perform anomaly detection for IaaS cloud computing platforms and determine whether the IaaS cloud computing platforms fail to run normally. However, it is challenging to effectively detect performance anomalies from a large amount of noisy and high dimensional performance metric data. In this paper, an efficient anomaly detection scheme is proposed for IaaS cloud computing platforms. The proposed scheme first designs a global locality preserving projection algorithm to perform feature extraction on performance metric data, and then introduces a local outlier factor algorithm to detect anomalies. A series of experiments are conducted on a private cloud computing platform. Experimental results show that our proposed global locality preserving projection algorithm outperforms the principal components analysis algorithm and the locality preserving projection algorithm and our proposed anomaly detection scheme is better than the state-of-the-art schemes for IaaS cloud computing platforms.
\end{abstract}

Keywords: Anomaly detection, IaaS cloud computing platform, Principal components analysis, Locality preserving projection, Local outlier factor

\section{Introduction}

Cloud computing is a new computing paradigm based on the Internet and has become a research focus [1]. With the continuous development of cloud computing, more and more enterprises choose to deploy their application systems on the IaaS cloud computing platform to improve the hardware resource utilization and then lower the IT operating cost [2]. However, as the number of application systems increases, the scale of IaaS cloud computing platform expands continuously and then the IaaS cloud computing platform becomes more and more complex. Increasing complexity will increase the probability that makes the IaaS cloud computing platform fail to run normally [3-4]. In order to monitor the running status of IaaS cloud computing platform, performance metric data are collected to perform anomaly detection for IaaS cloud computing platform and determine whether the IaaS cloud computing platform fails to run normally [5]. However, it is challenging to effectively detect anomalies from a large amount of noisy and high dimensional performance metric data [6].

In this paper, we propose an efficient anomaly detection scheme for IaaS cloud computing platform. The proposed anomaly detection scheme mainly consists of two steps, which are the feature extraction and anomaly detection. In the proposed anomaly detection scheme, a global locality preserving projection algorithm is first designed to perform feature extraction on performance metric data in order to reduce the dimension and improve the performance for anomaly detection, and then a local outlier factor algorithm is introduced to detect anomalies.

*Corresponding author 
A series of experiments are conducted on a private IaaS cloud computing platform built by using OpenStack and Xen open source software. Experimental results show that the proposed global locality preserving projection algorithm outperforms the principal components analysis algorithm and the locality preserving projection algorithm, moreover, the proposed anomaly detection scheme performs better than the state-of-the-art schemes for IaaS cloud computing platforms.

The remainder of this paper is organized as follows. Section 2 reviews existing works on feature extraction and anomaly detection. Section 3 presents our proposed anomaly detection scheme in detail. Performance evaluation is described in Section 4. Finally, conclusions are drawn in Section 5.

\section{Related work}

This section briefly reviews existing works on feature extraction and anomaly detection.

\subsection{Feature Extraction}

The performance metric data collected from IaaS cloud computing platform are noisy and high dimensional. In order to improve the performance of anomaly detection and shorten the processing time, feature extraction is often adopted to preprocess the performance metric data. The feature extraction is an important dimensionality reduction technology that can eliminate the noisy data and reduce the dimension of data. The principal components analysis (PCA) algorithm and the locality preserving projection (LPP) algorithm are two commonly used feature extraction algorithms. We will briefly review these two feature extraction algorithms as follows.

\subsubsection{Principal Components Analysis: Assume a data matrix} $X=\left[x_{1}, x_{2}, \cdots, x_{i}, \cdots, x_{n}\right] \in R^{D \times n}$, where $x_{i}$ is the $i$ th data sample and $n$ is the number of data sample in the data matrix. The data matrix $X$ is represented as follows.

$$
X=\left[x_{1}, x_{2}, \cdots, x_{i}, \cdots, x_{n}\right]=\left[\begin{array}{cccc}
x_{11} & x_{12} & \cdots & x_{n} \\
x_{21} & x_{22} & \cdots & x_{2 n} \\
\vdots & \vdots & \ddots & \vdots \\
x_{D 1} & x_{D 2} & \cdots & x_{D n}
\end{array}\right]
$$

Principal components analysis aims to find $d$ eigenvectors $w_{1}, w_{2}, \cdots, w_{d}$ to form the matrix $W=\left[w_{1}, w_{2}, \cdots, w_{d}\right]$ and uses the transformation $y_{i}=W^{T} x_{i}(i=1,2, \cdots, n)$ to obtain the new data matrix $Y=\left[y_{1}, y_{2}, \cdots, y_{i}, \cdots, y_{n}\right] \in R^{d}(d<D)$ that preserves the information from the original data matrix $X$ as much as possible and maximizes the variance of the new data matrix [7].

The variance of the new data matrix $Y$ is calculated as:

$$
J(W)_{P C A}=\sum_{i=1}^{n}\left\|y_{i}-y\right\|^{2}
$$

where $\bar{y}^{-}$is the mean of all $y_{i}$ that is calculated as:

$$
\bar{y}=\frac{1}{n} \sum_{j=1}^{n} y_{j}
$$

Then the variance of the new data matrix $Y$ can be represented as: 


$$
\begin{aligned}
J(W)_{P C A} & =\sum_{i=1}^{n}\left\|y_{i}-y\right\|^{2} \\
& =\sum_{i=1}^{n}\left\|y_{i}-\frac{1}{n} \sum_{j=1}^{n} y_{j}\right\|^{2} \\
& =\frac{1}{n^{2}} \sum_{i=1}^{n}\left\|n y_{i}-\sum_{j=1}^{n} y_{j}\right\|^{2} \\
& =\frac{1}{n^{2}} \sum_{i=1}^{n}\left\|\left(y_{i}-y_{1}\right)+\left(y_{i}-y_{2}\right)+\cdots+\left(y_{i}-y_{j}\right)+\cdots\left(y_{i}-y_{n}\right)\right\|^{2} \\
& =\frac{1}{n^{2}} \sum_{i=1}^{n}\left[\left(y_{i}-y_{1}\right)+\left(y_{i}-y_{2}\right)+\cdots+\left(y_{i}-y_{j}\right)+\cdots\left(y_{i}-y_{n}\right)\right] \\
& =\frac{1}{n^{2}} \sum_{i=1}^{n}\left[\left(y_{i}-y_{1}\right)+\left(y_{i}-y_{2}\right)+\cdots+\left(y_{i}-y_{j}\right)+\cdots\left(y_{i}-y_{n}\right)\right]^{2} \\
& =\frac{1}{n^{2}} \sum_{i=1}^{n}\left[\sum_{j=1}^{n}\left(y_{i}-y_{j}\right)\right]^{2}
\end{aligned}
$$

Because $y_{i}=W^{T} x_{i}$, the equation (4) can be calculated as:

$$
\begin{aligned}
J(W)_{P C A} & =\frac{1}{n^{2}} \sum_{i=1}^{n}\left[\sum_{j=1}^{n}\left(y_{i}-y_{j}\right)\right]^{2} \\
& =\frac{1}{n^{2}} \sum_{i=1}^{n}\left[\sum_{j=1}^{n}\left(W^{T} x_{i}-W^{T} x_{j}\right)\right]^{2} \\
& =W^{T} \frac{1}{n^{2}} \sum_{i=1}^{n}\left[\left(x_{i}-x_{1}\right)+\left(x_{i}-x_{2}\right)+\cdots+\left(x_{i}-x_{j}\right)+\cdots+\left(x_{i}-x_{n}\right)\right]^{2} W \\
& =W^{T} \frac{1}{n^{2}} \sum_{i=1}^{n}\left(n x_{i}-\sum_{j=1}^{n} x_{j}\right)^{2} W \\
& =W^{T} \sum_{i=1}^{n}\left(x_{i}-\frac{1}{n} \sum_{j=1}^{n} x_{j}\right)^{2} W
\end{aligned}
$$

Because $\bar{x}=\frac{1}{n} \sum_{j=1}^{n} x_{j}$, the equation (5) can be transformed as:

$$
\begin{aligned}
J(W)_{P C A} & =W^{T} \sum_{i=1}^{n}\left(x_{i}-\frac{1}{n} \sum_{j=1}^{n} x_{j}\right)^{2} W \\
& =W^{T} \sum_{i=1}^{n}\left(x_{i}-\bar{x}\right)^{2} W \\
& =W^{T} \sum_{i=1}^{n}\left(x_{i}-\bar{x}\right)\left(x_{i}-\bar{x}\right)^{T} W
\end{aligned}
$$

Let

$$
S_{P C A}=\sum_{i=1}^{n}\left(x_{i}-\bar{x}\right)\left(x_{i}-\bar{x}\right)^{T}
$$

where $S_{P C A}$ is covariance of the original data matrix $X$.

Then the equation (6) is transformed as:

$$
J(W)_{P C A}=W^{T} S_{P C A} W
$$


Therefore, solving the optimal data matrix $W$ that maximizes the variance of the new data matrix $Y$ can be transformed as the problem that solves the maximal value of the equation (8).

Because the matrix $W$ is a orthogonal matrix, the constraint condition for solving the maximal value of the equation (8) is $W^{T} W=E$, where $E$ is a identity matrix.

The Lagrange multiplier method is often used to solve the maximal value of the equation (8) by introducing a new variable $(\lambda)$ called a Lagrange multiplier and forming the Lagrange function as:

$$
\Lambda(W, \lambda)=W^{T} S_{P C A} W+\lambda\left(E-W^{T} W\right)
$$

(9)

Setting the partial derivative of $W$ to be zero as follows:

$$
\frac{\partial \Lambda(W, \lambda)}{\partial W}=S_{P C A} W-\lambda W=0
$$

Then the equation (10) can be transformed as:

$$
S_{P C A} W=\lambda W
$$

Therefore, the problem that solves the optimal matrix $W$ is transformed to be the problem that solves the eigenvalues of the covariance $S_{P C A}$.

$d$ eigenvectors $w_{1}, w_{2}, \cdots, w_{d}$ corresponding to the first $d$ biggest eigenvalues are selected and then the optimal matrix $W=\left[w_{1}, w_{2}, \cdots, w_{d}\right]$ can be obtained.

2.1.2. Locality Preserving Projection: The data processed by the principal components analysis can only preserve the global structure from the original data and loses the local structure. Existing research proves that manifold learning could find the low dimensional manifold structure hidden in the high dimensional data space. It is able to extract the local structure of the original data and makes the new data preserve the low dimensional manifold structure hidden in the high dimensional data space. In recent years, lots of manifold learning algorithms have been proposed, such as the isometric feature mapping, locally linear embedding, Laplacian eigenmaps, and so on. However, the nonlinear manifold learning algorithms show the high computation complexity. The locality preserving projection is a linear approximation of nonlinear Laplacian eigenmap and it inherits the capability that preserves the manifold structure from the nonlinear Laplacian eigenmap. It implements the linear transformation and reduces the computation complexity. It has been widely used in dimensionality reduction and pattern recognition [8].

The locality preserving projection aims to preserve the local manifold structure from the original data space as much as possible when reducing dimensionality. Therefore, the new data matrix $Y$ after linear transformation should minimize the following object function:

$$
J(W)_{L P P}=\sum_{i, j=1}^{n}\left\|y_{i}-y_{j}\right\|^{2} S_{i j}
$$

where the term $S_{i j}$ captures the adjacency relation between the points $x_{i}$ and $x_{j}$, the heat kernel is often used to calculate $S_{i j}$ as:

$$
S_{i j}= \begin{cases}e^{\left(-x_{i}-x_{j} \|^{2} / t\right)}, & \text { if } x_{i} \text { is among } k \text { nearest neighbors of } x_{j} \text { or } \\ & x_{j} \text { is among k nearest neighbors of } x_{i} \\ 0, & \text { otherwise }\end{cases}
$$

where $t$ is a parameter that is used to control the value of $S_{i j}$. The $k$-nearest neighbors algorithm is often introduced to determine the value of $S_{i j}$. 
Because $y_{i}=W^{T} x_{i}$, the equation (12) can be transformed as:

$$
\begin{aligned}
J(W)_{L P P} & =\sum_{i, j=1}^{n}\left\|y_{i}-y_{j}\right\|^{2} S_{i j} \\
& =\sum_{i, j=1}^{n}\left\|W^{T} x_{i}-W^{T} x_{j}\right\|^{2} S_{i j} \\
& =\sum_{i, j=1}^{n}\left(W^{T} x_{i}-W^{T} x_{j}\right)\left(W^{T} x_{i}-W^{T} x_{j}\right)^{T} S_{i j} \\
& =W^{T}\left[\sum_{i=1}^{n} \sum_{j=1}^{n}\left(x_{i}-x_{j}\right)\left(x_{i}-x_{j}\right)^{T} S_{i j}\right] W \\
& =W^{T}\left[\sum_{i, j=1}^{n}\left(x_{i} S_{i j} x_{i}^{T}-2 x_{i} S_{i j} x_{j}{ }^{T}+x_{j} S_{i j} x_{j}{ }^{T}\right)\right] W \\
& =W^{T}\left(2 \sum_{i=1}^{n} x_{i} D_{i i} x_{i}^{T}-2 \sum_{i, j=1}^{n} x_{i} S_{i j} x_{j}{ }^{T}\right) W \\
& =2 W^{T}\left(\sum_{i=1}^{n} x_{i} D_{i i} x_{i}^{T}-\sum_{i, j=1}^{n} x_{i} S_{i j} x_{j}{ }^{T}\right) W \\
& =2 W^{T}\left(X D X^{T}-X S X^{T}\right) W \\
& =2 W^{T} X(D-S) X^{T} W \\
& =2 W^{T} X L X^{T} W
\end{aligned}
$$

where $S$ is a symmetric matrix and $S_{i j}=S_{j i} . D$ is a diagonal matrix and its diagonal element $D_{i i}=\sum_{j=1}^{n} S_{i j}$. L is a Laplacian matrix and $L=D-S$.

Solving the optimal matrix $W$ is transformed to the problem that solves the minimal value for the equation (14). In order to avoid the influence of the arbitrary scaling factor, the locality preserving projection introduces a constraint condition as follows.

$$
W^{T} X D X^{T} W=1
$$

Therefore, the equation (15) is the constraint condition when solving the minimal value of the equation (14).

The minimal value of the equation (14) is solved by using the Lagrange multiplier method. A new variable $(\lambda)$ called the Lagrange multiplier is introduced and a Lagrange function is formed as:

$$
\Lambda(W, \lambda)=W^{T} X L X^{T} W+\lambda\left(1-W^{T} X D X^{T} W\right)
$$

Setting the partial derivative of $W$ to be zero as follows:

$$
\frac{\partial \Lambda(W, \lambda)}{\partial W}=X L X^{T} W-\lambda X D X^{T} W=0
$$

Then

$$
X L X^{T} W=\lambda X D X^{T} W
$$

Therefore, the problem that solves the optimal matrix $W$ is transformed to the problem that solves the eigenvalues via the equation (18).

The $d$ eigenvectors $w_{1}, w_{2}, \cdots, w_{d}$ corresponding to the first $d$ biggest eigenvalues is selected and then the optimal matrix $W=\left[w_{1}, w_{2}, \cdots, w_{d}\right]$ is obtained.

\subsection{Anomaly Detection}

Anomaly detection is an effective means that helps cloud computing administrators to monitor running status of cloud computing platforms and improves the dependability of cloud computing platforms. A number of works have been presented by collecting the 
performance metric data and analyzing the collected performance metric data in order to detect anomalies.

Wang et al. proposed the EbAT (Entropy-based anomaly testing) approach to detect anomalies by analyzing distributions for arbitrary metrics [9] [10]. They introduce entropy as a measurement to capture the degree of dispersal or concentration of such distributions. The resulting entropy distributions are aggregated to form entropy time series and then spike detecting, signal processing, or subspace methods are used to identify anomalies in entropy time series.

Pannu et al. proposed a self-evolving anomaly detection framework for developing highly dependable cloud platforms [11]. When the anomaly detection framework collects a new cloud performance data record, the anomaly detector calculates the anomaly score of this data record. If the anomaly score is higher than a defined threshold, the anomaly detector will send an anomaly warning to the management node in the cloud platforms. Then, the cloud platform administrator determines whether the anomaly is a real one or not. Finally, the anomaly detector learns results from the cloud platform administrator recursively and evolves in order to improve the detection performance.

Bhaduri et al. proposed an automated fault detection framework for cloud systems, which is called FDCS [12]. The proposed FDCS framework runs on the top of the distributed monitoring system, which is called Ganglia and collects the performance metric data of physical nodes by using the Ganglia system. Firstly, the FDCS framework introduces the distance-based anomaly score definition method to calculate the anomaly score of each physical node. Then, the FDCS framework sorts the physical nodes according to the anomaly score of each physical node and sends the sort list to the manage node in the cloud system. Finally, the system administrator checks the sort list and the failure parameter information of each physical node in the sort list in order to adopt effective prevention measurements to avoid that physical nodes fail.

Nguyen et al. proposed a black-box online fault localization system called FChain for cloud systems [13]. The proposed FChain system consists of two components, which are the FChain salve and the FChain master, respectively. The FChain slave is responsible for collecting the performance metric data, building the normal workload fluctuation model, and selecting the abnormal change point. If the service level objective (SLO) violations are detected, the FChain master checks each FChain slave in order to find out abnormal change point. Finally, the FChain master pinpoints faulty components according to the abnormal change propagation patterns and inter-component dependency relationships.

Smith et al. proposed an anomaly detection framework for autonomic management of compute cloud systems [14]. This framework consists of three main components, which are the data transformation, feature selection, and outlier detection, respectively. The data transformation is firstly used to reduce the problem of anomaly detection with different data types to the problem of finding outliers in a new space of a single data type. Then, the feature selection is performed to reduce the dimension of data for quick and better analysis. Finally, the outlier detection finds out and considers significant deviations as anomalies.

\section{Proposed Anomaly Detection Scheme}

In this section, we present an efficient anomaly detection scheme for IaaS cloud computing platform. The proposed anomaly detection scheme consists of two steps, which are the feature extraction and anomaly detection, respectively. Global locality preserving projection is first introduced to perform the feature extraction in order to reduce the dimension of performance metric data and improve the performance of anomaly detection. Then, a LOF-based anomaly detection algorithm is used to detect anomalies by analyzing the reduced performance metric data. 


\subsection{Global Locality Preserving Projection}

The principal components analysis uses the idea of maximizing variance in order to preserve the global structure from the original data. However, it cannot identify the low dimensional manifold structure hidden in the original data and the reduced data cannot preserve the local structure from the original data.

The locality preserving projection constructs a neighbor graph in order to represent the local neighborhood information and manifold structure in data. It can effectively identify the low dimensional manifold structure hidden in the original data. The reduced data can preserve the local neighbor information from the original data and then the locality preserving projection can extract the local structure from the original data. It can mark up the shortage of the principal components analysis. But it cannot extract the global structure from the original data.

In order to make the reduced data preserve the global and local manifold structure from the original data at the same time, this paper combines advantages of the principal components analysis and the locality preserving projection, and proposes an efficient global locality preserving projection. The proposed global locality preserving projection makes the reduced data preserve not only most variance information from the original data, but also the neighbor information from the original data. Therefore, the proposed global locality preserving projection can extract the global and local manifold structure from the original data.

If the data samples $x_{i}$ and $x_{j}$ satisfy the following relation:

$$
\left\|x_{i}-x_{j}\right\|^{2}<\varepsilon,(\varepsilon>0)
$$

Then, the data samples $x_{i}$ and $x_{j}$ are neighbors. Otherwise, they are not neighbors.

Therefore, the neighbor pair set of the original data can be described as:

$$
L^{\varepsilon}=\left\{(i, j)\left\|x_{i}-x_{j}\right\|^{2}<\varepsilon\right\}
$$

The non-neighbor pair set of the original data can be described as:

$$
G^{\varepsilon}=\left\{(i, j) \mid\left\|x_{i}-x_{j}\right\|^{2} \geq \varepsilon\right\}
$$

In order to capture the neighbor relations between data samples exactly, we construct an adjacency matrix $S$ where the element $S_{i j}$ can capture the neighbor relation between the data samples $x_{i}$ and $x_{j}$. The heat kernel used in the locality preserving projection is introduced to calculate the $S_{i j}$ as:

$$
S_{i j}= \begin{cases}\left.e^{-\left(\left|x_{i}-x_{j}\right|^{2} / t\right.}\right) & \text { if }(i, j) \in L^{\varepsilon} \\ 0, & \text { if }(i, j) \in G^{\varepsilon}\end{cases}
$$

The global locality preserving projection uses the sum of squares of the Euclidean distance between neighbors to capture the local manifold structure in the original data and then the local manifold structure can be represented as:

$$
J(W)_{L}=\sum_{(i, j) \in L^{s}}\left(y_{i}-y_{j}\right)^{2}
$$

Due to the adjacency matrix, the equation (23) can be transformed as:

$$
J(W)_{L}=\sum_{i=1}^{n} \sum_{j=1}^{n}\left(y_{i}-y_{j}\right)^{2} S_{i j}
$$

Through comparison, we can find that the equation (24) is the same to the equation (14). Therefore, the equation (24) can be transformed as: 


$$
\begin{aligned}
J(W)_{L}= & \sum_{i=1}^{n} \sum_{j=1}^{n}\left(y_{i}-y_{j}\right)^{2} S_{i j} \\
& =2 W^{T} X L X^{T} W
\end{aligned}
$$

The definition of $L$ in the equation (25) is the same to that in the locality preserving projection.

The global locality preserving projection uses the sum of squares of the Euclidean distance between non-neighbors to capture the global structure in the original data and then the global structure can be represented as:

$$
J(W)_{G}=\sum_{(i, j) \in G^{s}}\left(y_{i}-y_{j}\right)^{2}
$$

Because the set $G^{\varepsilon}$ is complement of the set $L^{\varepsilon}$, the equation (26) can be calculated as:

$$
\begin{aligned}
J(W)_{G} & =\sum_{(i, j) \in G^{e}}\left(y_{i}-y_{j}\right)^{2} \\
& =\sum_{i=1}^{n} \sum_{j=1}^{n}\left(y_{i}-y_{j}\right)^{2}-\sum_{(i, j) \in L^{e}}\left(y_{i}-y_{j}\right)^{2}
\end{aligned}
$$

Due to the equation (24), the equation (27) can be transformed as:

$$
\begin{aligned}
J(W)_{G} & =\sum_{i=1}^{n} \sum_{j=1}^{n}\left(y_{i}-y_{j}\right)^{2}-\sum_{(i, j) \in L^{e}}\left(y_{i}-y_{j}\right)^{2} \\
& =\sum_{i=1}^{n} \sum_{j=1}^{n}\left(y_{i}-y_{j}\right)^{2}-\sum_{i=1}^{n} \sum_{j=1}^{n}\left(y_{i}-y_{j}\right)^{2} S_{i j} \\
& =\sum_{i=1}^{n} \sum_{j=1}^{n}\left(1-S_{i j}\right)\left(y_{i}-y_{j}\right)^{2} \\
& =\sum_{i=1}^{n} \sum_{j=1}^{n}\left(1-S_{i j}\right)\left(W^{T} x_{i}-W^{T} x_{j}\right)^{2} \\
& =W^{T}\left[\sum_{i=1}^{n} \sum_{j=1}^{n}\left(1-S_{i j}\right)\left(x_{i}-x_{j}\right)\left(x_{i}-x_{j}\right)^{T}\right] W \\
& =W^{T}\left[\sum_{i=1}^{n} \sum_{j=1}^{n}\left(x_{i}-x_{j}\right)\left(x_{i}-x_{j}\right)^{T}-\sum_{i=1}^{n} \sum_{j=1}^{n} S_{i j}\left(x_{i}-x_{j}\right)\left(x_{i}-x_{j}\right)^{T}\right] W \\
& =W^{T}\left[\sum_{i=1}^{n} \sum_{j=1}^{n}\left(x_{i}-x_{j}\right)\left(x_{i}-x_{j}\right)^{T}\right] W-W^{T}\left[\sum_{i=1}^{n} \sum_{j=1}^{n} S_{i j}\left(x_{i}-x_{j}\right)\left(x_{i}-x_{j}\right)^{T}\right] W
\end{aligned}
$$

The equation (14) shows that

$$
\begin{aligned}
& W^{T}\left[\sum_{i=1}^{n} \sum_{j=1}^{n} S_{i j}\left(x_{i}-x_{j}\right)\left(x_{i}-x_{j}\right)^{T}\right] W \\
& =J(W)_{L L p} \\
& =2 W^{T} X L X^{T} W
\end{aligned}
$$

Then the equation (28) can be transformed as: 


$$
\begin{aligned}
J(W)_{G} & =W^{T}\left[\sum_{i=1}^{n} \sum_{j=1}^{n}\left(x_{i}-x_{j}\right)\left(x_{i}-x_{j}\right)^{T}\right] W-W^{T}\left[\sum_{i=1}^{n} \sum_{j=1}^{n} S_{i j}\left(x_{i}-x_{j}\right)\left(x_{i}-x_{j}\right)^{T}\right] W \\
& =W^{T}\left[\sum_{i=1}^{n} \sum_{j=1}^{n}\left(x_{i} x_{i}^{T}-2 x_{i} x_{j}^{T}+x_{j} x_{j}^{T}\right)\right] W-2 W^{T} X L X^{T} W \\
& =W^{T}\left[2 n \sum_{i=1}^{n} x_{i} x_{i}^{T}-2\left(\sum_{i=1}^{n} x_{i}\right)\left(\sum_{j=1}^{n} x_{j}^{T}\right)\right] W-2 W^{T} X L X^{T} W \\
& =2 W^{T}\left(n X X^{T}-X X^{T}\right) W-2 W^{T} X L X^{T} W \\
& =2 W^{T}\left[(n-1) X X^{T}-X L X^{T}\right] W
\end{aligned}
$$

The global locality preserving projection aims to make the reduced data preserve not only the global structure from the original data, but also the low dimensional manifold structure hidden in the original data. Therefore, the optimal matrix $W$ should minimize the $J(W)_{L}$ and maximize the $J(W)_{G}$ at the same time.

In order to avoid matrix inversion, the global locality preserving projection defines the objective function as follows:

$$
\begin{aligned}
J(W)_{G L P P}= & J(W)_{G}-J(W)_{L} \\
& =2 W^{T}\left[(n-1) X X^{T}-X L X^{T}\right] W-2 W^{T} X L X^{T} W \\
& =2 W^{T}\left[(n-1) X X^{T}-2 X L X^{T}\right] W
\end{aligned}
$$

Therefore, the problem solving the optimal matrix $W$ is converted to the problem solving the maximum value of the equation (31). In order to avoid the influence of the arbitrary scaling factor, the global locality preserving projection introduces a constraint condition as follows:

$$
W^{T} X D X^{T} W=1
$$

Therefore, the equation (32) is the constraint condition when solving the minimal value of the equation (31).

The minimal value of the equation (31) is solved by using the Lagrange multiplier method. A new variable $(\lambda)$ called the Lagrange multiplier is introduced and a Lagrange function is formed as:

$$
\Lambda(W, \lambda)=2 W^{T}\left[(n-1) X X^{T}-2 X L X^{T}\right] W+\lambda\left(1-W^{T} X D X^{T} W\right)
$$

Setting the partial derivative of $W$ to be zero as follows:

$$
\frac{\partial \Lambda(W, \lambda)}{\partial W}=2\left[(n-1) X X^{T}-2 X L X^{T}\right] W-\lambda X D X^{T} W=0
$$

Then

$$
X[2(n-1)-4 L] X^{T} W=\lambda X D X^{T} W
$$

Therefore, the problem that solves the optimal matrix $W$ is transformed to the problem that solves the eigenvalues via the equation (35).

The $d$ eigenvectors $w_{1}, w_{2}, \cdots, w_{d}$ corresponding to the first $d$ biggest eigenvalues is selected and then the optimal matrix $W=\left[w_{1}, w_{2}, \cdots, w_{d}\right]$ is obtained.

Then the new data matrix $Y$ can be calculated as:

$$
Y=\left[y_{1}, y_{2}, \cdots, y_{n}\right]=\left[W^{T} x_{1}, W^{T} x_{2}, \cdots, W^{T} x_{n}\right]
$$

The steps of the global locality preserving projection can be summarized as:

(1) Using the equation (19) to determine the neighbor relations between data samples in the original data, as well as obtain the neighbor pair set and the non-neighbor pair set.

(2) In order to capture the neighbor relations between data samples exactly, the heat kernel is introduced to calculate the neighbor relations between data samples and then the adjacency matrix $S$ is constructed. 
(3) The sum of squares of the Euclidean distance between neighbors in the original data is used to represent the local manifold structure of the original data and the sum of squares of the Euclidean distance between non-neighbors in the original data is used to represent the global structure of the original data.

(4) The objective function $J(W)_{G L P P}$ of the proposed global locality preserving projection is constructed.

(5) The Lagrange multiplier method is used to solve the maximum value of the objective function $J(W)_{G L P P}$ and the Lagrange function is constructed.

(6) Taking the derivative of the Lagrange function and obtaining the equation (35).

(7) Solving the eigenvalues of the equation (35). The $d$ eigenvectors corresponding to the first $d$ biggest eigenvalues is selected and then the optimal matrix $W$ is constructed.

\subsection{LOF-Based Anomaly Detection Algorithm}

The local outlier factor (LOF) is firstly proposed by Breunig et al. in 2000, which is a density-based outlier detection method [15]. The core idea of the local outlier factor is to assign each data object with a factor that captures its anomaly degree and determine whether the data object is an anomaly or not. This paper proposes an efficient LOF-based anomaly detection algorithm in order to detect anomalies.

The steps of our proposed LOF-based anomaly detection algorithm are described as follows:

(1) Calculating the $k$-distance of the data object $p$, namely $k$-distance $(p)$, which is defined as the distance $d(p, q)$ between the data objects $p$ and $q$ if the data object $q$ satisfies the following two conditions:

(1.1) for at least $k$ data objects $q^{\prime} \in D \backslash\{p\}$, it holds that $d\left(p, q^{\prime}\right) \leq d(p, q)$;

(1.2) for at most $k-1$ data objects $q^{\prime} \in D \backslash\{p\}$, it holds that $d\left(p, q^{\prime}\right)<d(p, q)$.

The Euclidean distance between two data objects are used to calculate the distance $d(p, q)$ between two data objects $p$ and $q$.

(2) Calculating the $k$-distance neighborhood of the data object $p$.

Given the $k$-distance of the data object $p$, its $k$-distance neighborhood is the set of data objects whose distance from $p$ is not greater than the $k$-distance of the data object $p$, namely

$$
K N N(p)=N_{k}(p)=\{q \in D \backslash\{p\} \mid d(p, q) \leq k-\operatorname{distance}(p)\}
$$

These data objects $q$ are the $k$-nearest neighbors of the data object $p$ and from the $k$ distance neighborhood of the data object $p$.

(3) Calculating the reachability distance of the data object $p$ w.r.t the data object $q$.

The reachability distance of the data object $p$ with respect to the data object $q$ is defined as:

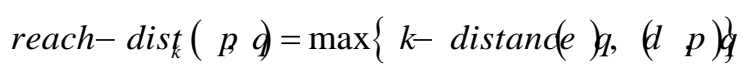

(4) Calculating the local reachability density of the data object $p$.

The local reachability density of the data object $p$ is defined as:

$$
\operatorname{lrd}_{k}(p)=1 /\left[\frac{\sum_{q \in N_{k}(p)} \text { reach-dist } t_{k}(p, q)}{\left|N_{k}(p)\right|}\right]
$$

(5) Calculating the local outlier factor of the data object $p$.

The local outlier factor of the data object $p$ is defined as: 


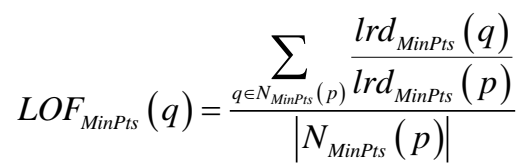

(6) The local outlier factor of each data object is used to determine that whether the data object is an outlier or not. If its local outlier factor is approximately 1 , it indicates that this data object cannot be identified as an anomaly. If the local outlier factor of the data object is much higher than 1 , it indicates that this data object is an anomaly.

\section{Performance Evaluation}

In this section, we first describe the experiment environment and then the experimental results are shown.

\subsection{Experiment Environment}

The experiment test bed consists of six physical nodes, which are connected with the Gigabit Ethernet. One physical node is selected as the cloud controller, while the rest are deployed as the compute node. The open source software OpenStack [16] is used to build a simple private OpenStack cloud computing platform, which adopts the Xen 3.1.0 [17] to implements the virtualization. Four virtual machines run on each physical node.

The distributed online service benchmark RUBiS is first deployed on the OpenStack cloud computing platform [18]. Then, 50 faults are injected into the OpenStack cloud computing platform. Finally, real time performance metric data are collected from the OpenStack cloud computing platform and are analyzed.

The precision, recall, F-measure, and average computational overhead are introduced to evaluate the performance of the proposed global locality preserving projection and anomaly detection scheme.

Let $T P$ (True Positive) to be a performance metric data set where performance metric data are abnormal and they are also detected as abnormal. Let FP (False Positive) to be a performance data set where performance metric data are normal but they are detected as abnormal. Let $F N$ (False Negative) to be a performance metric data set where performance metric data are abnormal but they are detected as normal. Let $T N$ (True Negative) to be a performance metric data set where performance data are normal and they are also detected as normal. Then, the precision, recall, F-measure can be defined as follows.

$$
\begin{gathered}
\text { Precision }=\frac{|T P|}{|T P|+|F P|} \\
\text { Recall }=\frac{|T P|}{|T P|+|F N|} \\
F-\text { measure }=\frac{2 \times \text { Precision } \times \text { Recall }}{\text { Precision }+ \text { Recall }}
\end{gathered}
$$

A series of experiments are conducted to compare our proposed global locality preserving projection with the principal components analysis and locality preserving projection, as well as our proposed anomaly detection scheme with existing anomaly detection schemes, such as EbAT, FDCS, and FChain that have been described in the Section 2.2.

\subsection{Experimental Results}

In this section, the experimental results are presented. 


\section{Table 1. Precision, Recall, and F-Measure for Different Anomaly Detection Schemes}

\begin{tabular}{|c|c|c|c|c|c|}
\hline $\begin{array}{c}\text { Anomaly detection } \\
\text { schemes }\end{array}$ & $|T P|+|F P|$ & $|T P|$ & Precision & Recall & F-measure \\
\hline No-Extraction+LOF & 52 & 43 & $82.7 \%$ & $86 \%$ & $84.3 \%$ \\
\hline PCA+LOF & 48 & 42 & $87.5 \%$ & $84 \%$ & $85.7 \%$ \\
\hline LPP+LOF & 49 & 45 & $91.8 \%$ & $90 \%$ & $90.9 \%$ \\
\hline GLPP+LOF & 50 & 48 & $96 \%$ & $96 \%$ & $96 \%$ \\
\hline EbAT & 53 & 46 & $86.8 \%$ & $92 \%$ & $89.3 \%$ \\
\hline FDCS & 52 & 45 & $86.5 \%$ & $90 \%$ & $88.2 \%$ \\
\hline FChain & 51 & 41 & $80.4 \%$ & $82 \%$ & $81.5 \%$ \\
\hline
\end{tabular}

Table 1 shows the precision, recall and F-measure for different anomaly detection schemes. It can be seen that PCA+LOF, LPP+LOF, GLPP+LOF show higher precisions than other anomaly detection schemes without feature extraction algorithms and our proposed anomaly detection scheme GLPP+LOF is better than the rest schemes in terms of precision, recall, and F-measure. That is because the feature extraction algorithms can reduce data noise and data fluctuation, as well as preserve most useful data information, in order to improve precision. GLPP can extract the global and local manifold structure form the original data. Therefore, GLPP performs better than PCA and LPP in terms of precision, recall, and F-measure.

Table 2. Average Computational Overhead for Different Anomaly Detection Schemes

\begin{tabular}{|c|c|}
\hline Anomaly detection schemes & Average Computational Overhead (s) \\
\hline No-Extraction+LOF & 1.0216 \\
\hline PCA+LOF & 0.1202 \\
\hline LPP+LOF & 0.2289 \\
\hline GLPP+LOF & 0.2452 \\
\hline EbAT & 0.8569 \\
\hline FDCS & 0.9872 \\
\hline FChain & 0.7976 \\
\hline
\end{tabular}

Table 2 shows average computational overhead for different anomaly detection schemes. It can be seen that PCA+LOF, LPP+LOF, GLPP+LOF consume less average computational overhead than other anomaly detection schemes without feature extraction algorithms. That is because feature extraction algorithms can reduce the data noise and data fluctuation, average computational overhead will be reduced greatly. Although GLPP+LOF has higher average computational overhead than PCA+LOF and LPP+LOF, its average computational overhead is less than 0.25 .

\section{Conclusions}

An efficient anomaly detection scheme is proposed to monitor the running status of IaaS cloud computing platforms in this paper. The proposed anomaly detection scheme proposes a global locality preserving projection algorithm to perform feature extraction on performance metric data, and then introduces a local outlier factor algorithm to detect anomalies. A series of experiments are conducted on a private cloud computing platform that is built by using the Xen and OpenStack open source software. Experimental results show that our proposed global locality preserving projection algorithm performs better than the principal components analysis algorithm and the locality preserving projection algorithm, moreover, our proposed anomaly detection scheme is superior to existing schemes. 


\section{Acknowledgments}

The work of this paper is supported by the National Natural Science Foundation of China under Grant No. 61502102, No. 61370078, and No. 61402109, Fujian Province Education Scientific Research Projects for Young and Middle-aged Teachers under Grant No. JA15122, National Undergraduate Training Programs for Innovation and Entrepreneurship under Grant No. 201510394021, and Fujian Normal University Undergraduate Training Programs for Innovation and Entrepreneurship under Grant No. cxxl-2015163.

\section{References}

[1] A. N. Toosi, R. N. Calheiros, R. Buyya, "Interconnected cloud computing environments: Challenges, taxonomy, and survey", ACM Computing Surveys, vol. 47, no. 1, (2014), Article 7.

[2] Y. Jiang, B. Zhao, S. Wang, D. Sun, "Research of enterprise private cloud computing platform based on openstack", International Journal of Grid and Distributed Computing, vol. 7, no. 5, (2014), pp. 171-180.

[3] Q. Guan, S. Fu, N. D. Bardeleben, S. Blanchard, "Exploring time and frequency domains for accurate and automated anomaly detection in cloud computing systems", Proceedings of 2013 IEEE 19th Pacific Rim International Symposium on Dependable Computing, (2013), 196-205.

[4] Q. Guan, S. Fu, "Wavelet-based multi-scale anomaly identification in cloud computing systems”, 2013 IEEE Global Communications Conference, (2013), 1379-1384.

[5] Q. Guan, S. Fu, "Auto-AID: A data mining framework for autonomic anomaly identification in networked computer systems", 2010 IEEE 29th International Performance Computing and Communications Conference, (2010), 73-80.

[6] S. Fu, "Performance metric selection for autonomic anomaly detection on cloud computing systems", 2011 IEEE Global Telecommunications Conference, (2011), 1-5.

[7] L. I. Kuncheva, W. J. Faithfull, "PCA feature extraction for change detection in multidimensional unlabeled data", IEEE Transactions on Neural Networks and Learning Systems, vol. 25, no. 1, (2014), 69-80.

[8] X. He, D. Cai, W. Min, "Statistical and computational analysis of locality preserving projection", Proceedings of the 22nd International Conference on Machine Learning, (2005), 281-288.

[9] C. Wang, K. Schwan, M. Wolf, "EbAT: An entropy based online anomaly tester for data center management", 2009 IFIP/IEEE International Symposium on Integrated Network Management-Workshops, (2009), 79-80.

[10] C. Wang, V. Talwar, K. Schwan, P. Ranganathan, "Online detection of utility cloud anomalies using metric distributions", Proceedings of the 2010 IEEE/IFIP Network Operations and Management Symposium, (2010), 96-103.

[11] H. S. Pannu, J. Liu, S. Fu, "A self-evolving anomaly detection framework for developing highly dependable utility clouds", 2012 IEEE Global Communications Conference, (2012), 1605-1610.

[12] K. Bhaduri, K. Das, B. L. Matthews, "Detecting abnormal machine characteristics in cloud infrastructures", Proceedings of 11th IEEE International Conference on Data Mining Workshops, (2011), 137-144.

[13] H. Nguyen, Z. Shen, Y. Tan, X. Gu, "FChain: Toward black-box online fault localization for cloud systems", Proceedings of 2013 IEEE 33rd International Conference on Distributed Computing Systems, (2013), 21-30.

[14] D. Smith, Q. Guan, S. Fu, “An anomaly detection framework for autonomic management of compute cloud systems", Proceedings of 34th Annual IEEE International Computer Software and Applications Conference Workshops, (2010), 376-381.

[15] M. M. Breunig, H. P. Kriegel, R. T. Ng, J. Sander, "LOF: identifying density-based local outliers", Proceedings of the ACM SIGMOD International Conference on Management of Data, vol. 29, no. 2, (2000), 93-104.

[16] A. Corradi, M. Fanelli, L. Foschini, "VM consolidation: A real case based on OpenStack Cloud", Future Generation Computer Systems, vol. 32, no. 1, (2014), 118-127. 
[17] D. Williams, H. Jamjoom, H. Weatherspoon, "The Xen-Blanket: Virtualize once, run everywhere", Proceedings of the EuroSys 2012 Conference, (2012), 113-126.

[18] E. Cecchet, J. Marguerite, W. Zwaenepoel, "Performance and scalability of EJB applications", Proceedings of the 17th ACM SIGPLAN conference on Object-oriented programming, systems, languages, and applications, (2002), 246-261.

\section{Authors}

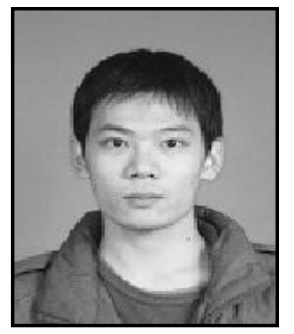

Mingwei Lin, he received his B.S. and Ph.D. degrees from Chongqing University, China, in July 2009 and December 2014. Currently, he is a lecturer in the Faculty of Software, Fujian Normal University, China. His research interests include anomaly detection, NAND flash memory, Linux operating system, and cloud computing. He got the CSC-IBM Chinese Excellent Student Scholarship in 2012.

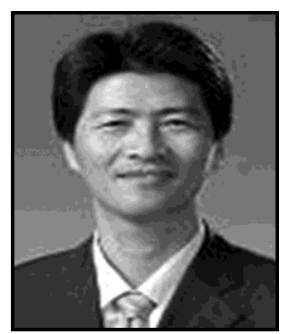

Zhiqiang Yao, he received the $\mathrm{PhD}$ degree from Xidian University, China, in 2014. Currently, he is a professor in the Faculty of Software, Fujian Normal University, ACM Professional Membership, Senior Member of China Computer Federation (CCF). His current research interests mainly focus on security in cloud computing, multimedia security.

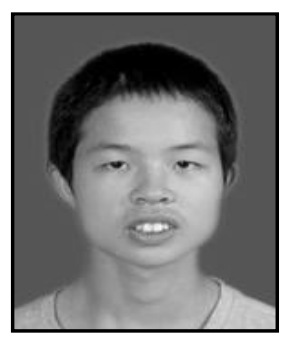

Fei Gao, he is a junior student in the Faculty of Software, Fujian Normal University, Fuzhou, China. He majors in Software Engineering. He has applied for a National Undergraduate Training Program for Innovation and Entrepreneurship successfully. His current research interests include cloud computing, android application development, and flash memory.

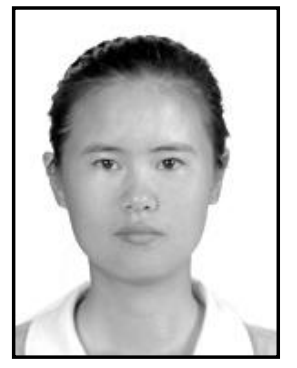

Yang Li, she is a junior student in the Faculty of Software, Fujian Normal University, Fuzhou, China. Her major is Software Engineering. She is hosting a Fujian Normal University Undergraduate Training Program for Innovation and Entrepreneurship. Her current research interests include anomaly detection, cloud computing, and flash memory. 